\title{
On the Existence of a Semi-permeable Membrane enclosing the Seeds of some of the Gramineae.
}

BY

ADRIAN J. BROWN.

URING a recent investigation of the conditions governing the absorption of water by the ripe grain of certain cereals, some experiments with the grain of Hordeum vulgare, var. caerulescens, indicated that the embryo and endosperm of the grain are contained within a semi-permeable covering.

The grain of this variety of $H$. vulgare is characterized by a greenishblue colour, due to the presence of a blue pigment in its aleurone cells. As the colour of this pigment, like litmus, is changed to red by acid, the grain is therefore naturally furnished with an indicator by means of which the access of free acid to its aleurone cells may be detected.

During some experiments with the blue variety of $H$. vulgare, in which the grain was steeped in a dilute solution (I per cent.) of sulphuric acid, it was noticed that when the skins of the grain were punctured or otherwise damaged the colour of the aleurone cells of the grain adjacent to the damaged part of the skin rapidly changed to red, and that this colour transformation gradually spread to all the cells of the aleurone layer, demonstrating diffusion of acid throughout the endosperm of the grain. When, however, undamaged grain of the same variety of barley was steeped in a similar solution of acid it was observed that although the contents of the grain became soft and swollen from absorption of water, the aleurone cells retained their original blue colour, indicating that the acid had not obtained access to the interior of the grain. It was further noticed that this condition remained for a week or even longer, if germination of the grain was prevented or delayed by keeping it submerged in the acid steeping liquid. If, however, germination took place, with consequent rupture by the growing roots of the skins of the grain where they protect the base of the embryo, the aleurone cells of the grain speedily changed to a red colour, indicating that acid had diffused through the tissues of the embryo into the endosperm of the seed.

[Annals of Botany, Vo1. XXI. No. LXXXI. January, 1907.] 
These observations regarding the behaviour of the grain of the blue variety of $H$. vulgare when steeped in dilute sulphuric acid therefore indicated that water alone obtains entrance to the grain under these conditions if its coverings remain intact, and that consequently the coverings of the grain behave as a semi-permeable or selective membrane with regard to an aqueous solution of sulphuric acid. But as this conclusion was based solely on indications furnished by the colour reaction with acid of the blue pigment of the aleurone cells of the grain, it was evidently desirable to test it by other methods of investigation.

In further experiments grains of the blue variety of $H$. vulgare were steeped in a I per cent. solution of sulphuric acid for periods of time varying from 24 to 72 hours, and subsequently were cut-some longitudinally, and others transversely-into sections. On testing the reaction of these sections with a solution of methyl-orange no indication of the presence of free acid was obtained in any portion of the seed within the testa-the coverings of the grain, however, exhibited an acid reaction. In all the experiments the presence of water was demonstrated within the seed coverings, both by the swollen and soft condition of the endosperm and also by the turgid condition of the cells of the embryo.

It was evident, however, that more conclusive proof that the coverings of the grain functioned as a semi-permeable envelope would be obtained, if, following steeping the grain in a solution of sulphuric acid, concentration of the solution of acid was demonstrated-for manifestly concentration must take place if water only is absorbed by the grain under these conditions.

Experiments with barley grains, in which they were steeped in normal ( 4.9 per cent.) sulphuric acid, and the remaining acid solution was subsequently titrated with standard alkali, readily demonstrated that a marked concentration of acid in the solution takes place under these conditions. But it appeared more satisfactory to establish, if possible, that the concentration which takes place is proportional to the volume of water abstracted from the solution of acid by the barley grains during steeping.

The following experiment was made with this object in view. 10.899 grms. of air-dried barley grains were in the first instance steeped in 15 c.c. normal (4.9 per cent.) sulphuric acid for 3 hours, and afterwards the grains were drained, well dried in filter-paper, and reweighed. The weight obtained was 12.509 grms. This preliminary treatment of the grain was carried on in the first instance to neutralize a feeble basic condition apparently natural to the skins of the grain, and secondly, in order to correct as nearly as possible for the weight of moisture adhering to the skins of the grain after draining and drying in filter-paper.

Following this preliminary treatment the grain was again steeped in I5 c.c. normal sulphuric acid and allowed to remain for 48 hours. 
Subsequently after draining and drying the grain in filter-paper as before it was found to weigh $14.459 \mathrm{grms}$. The grain had therefore absorbed I. $95^{\circ}$ grms. of moisture from the normal acid in which it had been steeped. This weight would represent $\mathrm{I} \cdot 95^{\circ}$ c.c. water abstracted from the original volume of normal acid if the skins of all the grains of barley employed were undamaged and had permitted the passage of water only through them; but on examination it was found that the skins of $7 \cdot 2$ per cent. of the grains employed had been injured and had admitted the passage of both acid and water to the interior of the grains. Consequently the volume of pure water abstracted from the acid solution and tending to concentrate it is $\mathrm{I} \cdot 8 \mathrm{I}$ c.c. $(\mathrm{I} \cdot 95$ c.c. $-0 . \mathrm{I} 4$ c.c.). On subtracting this amount from the original volume of 15 c.c. of normal acid employed, I3. I9 c.c. remain as the diminished volume of the acid solution calculated from the weight of water absorbed by the grain.

On directly titrating Io c.c. of the acid solution with normal ( 4.0 per cent.) caustic soda, it was found that II.I c.c. were required for neutralization ; hence II.I c.c. of the original normal acid have been concentrated to a volume of Io c.c., which is equivalent to a concentration of the original I5 c.c. employed to $13.5^{\text {I c.c. }}$

Therefore, from the weight of water absorbed by the grain, a concentration of the original 15 c.c. of normal acid to 13.19 c.c. has been found, and by direct titration a concentration of $13.5 \mathrm{I}$ c.c.-close agreement considering the relatively large errors present in the method of experiment which it was found necessary to adopt.

In order to demonstrate still more conclusively that a solution of sulphuric acid is concentrated by the semi-permeable property of barley grains, four separate quantities of grain were steeped successively for 24 hours each in the same solution of normal sulphuric acid in order to intensify the concentration effect. On subsequently titrating the acid solution which remained unabsorbed by the grain its strength was found to have increased from the original concentration of 4.9 per cent. to 7.62 per cent. $\mathrm{H}_{2} \mathrm{SO}_{4}$.

The experiments described demonstrate conclusively that a separation of water and sulphuric acid is effected when undamaged grains of barley are steeped in solutions of sulphuric acid not exceeding a concentration of 4.9 per cent. As it appeared desirable, however, to ascertain if a similar phenomenon is exhibited when barley grains are steeped in more concentrated acid, some grains of the blue variety of $H$. vulgare were immersed in solutions of $9 \cdot 0,18 \cdot 0$, and $3^{6 \cdot 0}$ per cent. $\mathrm{H}_{2} \mathrm{SO}_{4}$ for 44 hours.

On subsequent examination it was found in all the experiments that the original blue colour of the undamaged grains remained unchanged. Further examination by means of methyl-orange also demonstrated that no free acid had penetrated to the interior of the grains. When the grains 
were cut, however, a difference was observed in the conditions of their contents in respect of the amount of water absorbed during steeping in the solutions of acid. The endosperms of the grains steeped in 9 per cent. sulphuric acid were soft, and the tissues of the embryos were turgid through absorption of water. The endosperms of the grains steeped in 18 per cent. acid were not so soft, and the embryos, although distinctly moist, were much less so than in the experiment with weaker acid. In the case of the grains steeped in $3^{6}$ per cent. acid, both their endosperms and embryos appeared to be as hard and dry as they were previous to steeping in the acid.

It is evident when a semi-permeable membrane capable of separating water and sulphuric acid is in action some such results as those described are to be expected, and they suggest some interesting questions in connexion with osmosis which are reserved for future investigation.

In order to obtain additional proof that solutions of sulphuric acid of such high concentration as 18 and $3^{6}$ per cent., employed in the experiments just described, had not gained access to the embryos of the barley grains and destroyed their vitality, some of the grains which had been steeped in these two solutions of acid were well washed in water and subsequently placed under conditions favourable for germination. In a few days all were in vigorous growth.

Following on the experiments with sulphuric acid which have been described, some experiments were made in which barley grains were steeped in solutions of various salts in order to ascertain if water alone passes into the interior of the grains under these conditions.

5 per cent. solutions of cupric sulphate, ferrous sulphate, potassium chromate, silver nitrate, and potassium ferrocyanide, were employed with the result that no trace of any of these salts was found within the coverings of the grains after three days' steeping, although in all cases the embryos and endosperms of the grains were softened and distended with water.

Experiments were also made with solutions of sodium hydrate of varying concentration. Solutions of I per cent. strength and upwards destroyed the coverings of the grain and ultimately disintegrated the grain completely.

The coverings of the grain were, however, found to withstand the action of $\mathrm{a} 0.5$ per cent. solution, and under these conditions, although water passed from the solution into the grain, sodium hydrate was excluded.

Experiments with solutions of hydrochloric acid of normal (3.65 per cent.) strength also showed that this acid, like sulphuric acid, is excluded from the interior of the grains.

With nitric acid, however, somewhat different results were obtained. When barley grains were steeped in a I per cent. solution of this acid it was found that the acid was excluded during 24 hours' immersion, and in 
the case of a small number of the grains a similar result was obtained after 48 hours' steeping, but eventually it was found that all the grains admitted acid ${ }^{1}$.

A similar condition was noticed, but after a much shorter interval of time, when the grains were immersed in 5 per cent. nitric acid. From the appearance of the grains after steeping, there is reason, however, to believe that the passage of nitric acid to the interior of the grains does not result from a selective action of their semi-permeable envelope, but from destruction of the semi-permeable property of the envelope by the chemical action of the acid.

Apparently, however, an instance of the envelope exhibiting a power of selection for matter other than water is found in the passage of iodine into the barley grain.

When barley grains are steeped in a I per cent. solution of iodine in iodide of potassium ( 5 per cent.) solution for 24 hours, the passage of iodine through the envelope into the interior of the grains is evidenced by the contents of the cells of the embryo and of the aleurone cells being stained brown, and also more markedly by the starch granules of the starchcontaining cells of the endosperm being stained blue.

The latter phenomenon, which renders it easy to study the manner in which iodine passes into the grain, indicates that it penetrates all parts of the skins enveloping the endosperm at approximately the same velocity, with the exception of the part in the neighbourhood of the ventral-furrow, through which it appears to pass with difficulty.

In the first instance it seemed probable that access of iodine to the interior of the grain was only obtained after destruction of the semipermeable property of the envelope by the chemical action of the iodine, but experiments in which grains of barley previously stained with iodine were steeped in a solution of sodium hyposulphite, appeared to show that such is not the case. Under such condition, if the semi-permeable character of the envelope of the grains was destroyed, the sodium hyposulphite would diffuse into the grains and discolour the starchcontaining cells of their endosperms which had previously been coloured with iodine; and, in confirmation of this, direct experiment showed that when grains of barley stained with iodine were steeped in a solution of sodium hyposulphite after their skins were punctured or otherwise damaged, the sodium hyposulphite diffused into the grains and discoloured them. On the other hand, when iodine-stained grains with uninjured skins were steeped in a solution of sodium hyposulphite, no discoloration was observed even after the grains had been immersed in the solution for five days.

${ }^{1}$ It appeared that entrance of the acid was always obtained at the germ, or proximal, end of the seed. 
The experiments described above demonstrate that the embryo and endosperm of the barley grain are enclosed within an envelope through which water and iodine can pass to the interior of the grain, but through which certain acids and salts cannot pass. As there appeared to be no known instance of the occurrence in the vegetable kingdom of a membrane other than one of living protoplasm, which possessed a marked semipermeable property, it seemed very desirable to inquire if the semipermeable property of the envelope of the barley grain was a function of living protoplasm, although from the first it appeared very improbable that it was so when the conditions ruling in some of the experiments already described were considered.

In order to demonstrate the semi-permeable character of living protoplasm by plasmolysis, solutions either of inert salts, or of such bodies as cane sugar, which do not exert an injurious action on the protoplasm of the living cell experimented with, must be employed, for otherwise, as the solute must come into direct contact with the protoplasm of the cell in order to exert its osmotic influence, its vitality would be destroyed and it would cease to function as a semi-permeable membrane. But in some of the previous experiments it has been shown that the semi-permeable property of the envelope of the barley grain is exhibited with solutions of sulphuric acid of very high concentration, and it appears inconceivable that such strong acid can come in contact with living protoplasm without destroying its vitality at once. Moreover it has been shown that when solutions of such poisons for protoplasm as silver nitrate and cupric sulphate are employed these salts are excluded from the interior of the barley grain. Additional evidence that living protoplasm does not act as the semipermeable membrane of the barley grain is also furnished by the results of the experiments with iodine, which have already been described. In these experiments it was shown that iodine penetrates the coverings of the grain, and yet on subsequent immersion of the grain in a solution of sodium hyposulphite the coverings through which iodine has already passed prevent the passage of the salt. To regard the selective character of the coverings as depending on the action of living protoplasm after the passage of a strong poison like iodine through them seems unreasonable.

Conclusive proof that the semi-permeable character of the envelope surrounding the grain does not depend on the activity of living protoplasm was, however, obtained by experiments conducted with grains of barley after they had been subjected to the action of boiling water. Some barley grains were immersed in boiling water, and at intervals of $5,10,30,60$, and 120 minutes a number of the grains were removed. On examination it was found that the skins of all the grains which had been steeped in boiling water for 120 minutes were ruptured and their contents were extruding, and of those grains similarly treated for 60 minutes all but one 
were ruptured. In the case of the grains boiled for 30 , 10, and 5 minutes all remained whole.

Following on the treatment with boiling water the grains from the three last experiments, the skins of which were apparently uninjured, were steeped in normal sulphuric acid for 48 hours. On subsequent examination it was found that no trace of acid had penetrated to the interior of the grains except in a very few instances in which the skins of the grains had been accidentally damaged. In order to obtain additional evidence that life in the grains had been destroyed by the treatment to which they had been subjected, some of the grains which had been immersed in boiling water for the shortest period of time ( 5 minutes) were placed under conditions favourable to germination, with the result that they exhibited no signs of life.

The above experiments therefore show conclusively that the semipermeable property of the envelope of the barley grain is not a function of living protoplasm.

A study of the nature and position of the semi-permeable envelope enclosing the barley grain was then commenced. Previous observations regarding the behaviour of the blue pigment in the aleurone cells of grains of $H$. vulgare, var. caerulescens, when steeped in an acid solution, demonstrate that the semi-permeable envelope must occupy a position which is external to the aleurone cells of the endosperm, and also that it must enclose both the embryo and endosperm of the grain.

The envelope enclosing the embryo and endosperm of the barley grain apparently consists ${ }^{1}$ : (I) of the pales, originally the floral envelope ; (2) of the pericarp, composed of several layers derived from the component parts of the walls of the ovary; and (3) of the spermoderm, composed of the ovular integuments together with the outer layer of the nucellar tissue. The semi-permeable property of the grain therefore should be located in one or more of these coverings.

The pales of the grain do not function as the semi-permeable covering, for after their removal the grain exhibits its semi-permeable property equally as well as before. The property also does not appear to be a function of any part of the pericarp, for the layers of cells composing this covering are disintegrated when the barley grain is digested in a $3^{6}$ per cent. solution of sulphuric acid without the semi-permeability of the grain being destroyed. (See above, p. 8I.)

The spermoderm of the grain, however, resists the action of $3^{6}$ per cent. sulphuric acid in a very remarkable manner, and hence this covering or one of its component layers probably constitutes the semi-permeable membrane of the barley grain.

'See 'Développement et constitution de l'endosperme de l'orge'. W. Johannsen. Comptes Rendus des travaux du Laboratoire de Carlsberg. Vol. 2, I884, 63 . 
In order to investigate this point further, grains of $H$. vulgare, var. cacrulescens, and of several varieties of $H$. distichum were steeped for 48 hours in solutions of methyl-violet and of fuchsin, and also in solution of various salts which could be readily traced by micro-chemical means, and subsequently sections of the grains were prepared for the purpose of studying microscopically the limit of passage of the reagents employed through the coverings of the grains.

The best results were obtained with grains which were in the first instance steeped in a 3 per cent. solution of silver nitrate for 48 hours, and afterwards steeped for 48 hours in a 5 per cent. solution of sodium chloride. By this means the limit of penetration of the silver nitrate through the skins of the grain was rendered visible by its precipitation as silver chloride in the cellular tissues and subsequent blackening on exposure to light.

Microscopic examination of sections of barley grains treated in this manner demonstrates that silver nitrate passes through the pales and pericarp into the spermoderm, which, in part at least, is coloured by the silver salt. A thin layer of uncoloured membrane, however, remains between the stained portion of the spermoderm and the walls of the aleurone cells of the endosperm. Probably this layer of the spermoderm is derived from the epidermis of the nucellus, but its identity has not been satisfactorily determined at present.

When transverse sections of a barley grain treated with silver nitrate and sodium chloride are examined in the neighbourhood of the funicle a very well-defined line is found, indicating that the silver salt, after penetrating the spermoderm some distance, stops abruptly at the outermost layer of the sheaf-like mass of empty cells, which are usually regarded as the remains of the cells of the nucellus; but it appears undesirable to draw any definite conclusion from this observation owing to the complex character of the envelope of the barley grain in the neighbourhood of the funicle.

There seems to be no doubt that the semi-permeable property of the barley grain centres in the spermoderm, but whether the property is confined to one of its layers only has not been definitely determined as yet.

As it appeared interesting to ascertain if the grain of other of the Gramineae than Hordeum exhibited the phenomenon of semi-permeability, grains of Avena, Triticum, and Secale were steeped in a normal solution ( 4.9 per cent.) of sulphuric acid for 48 hours, and subsequently examined. It was found that they all evidenced the possession of a semi-permeable covering, as in the case of Hordeum. In the case of Triticum and Secale, however, a much larger proportion of damaged grains not capable of excluding acid were found as compared with Hordeum and Avena. 
Apparently this is due to the grain of Triticum and Secale not retaining the pales as a protective covering, as in the case of Hordeum and Avena, and consequently to the skins which compose the pericarp and spermoderm of the grain of Triticum and Secale being more exposed to accidental injury.

\section{CONCLUSION.}

This paper gives an account of the preliminary work of an investigation which is being continued, and although the results so far obtained suggest further discussion both from a botanical and a physico-chemical point of view, it appears desirable at present to await the results of experimental work which is proceeding. The following summary appears, however, to be justified :-

I. The grain of Hordeum is enclosed within a semi-permeable or selective covering, which permits the passage of water and iodine to the interior of the grain, but which prevents the passage of sulphuric and hydrochloric acids, and all salts of metals at present examined, when they are in aqueous solution.

2. The semi-permeable property of the covering of the grain of Hordeum is not due to the action of living protoplasm.

3. The semi-permeable property of the covering of the grain of Hordeum is located in the spermoderm of the grain.

4. The grain of Avena, Triticum, and Secale is enclosed in a semipermeable covering apparently similar to that of the grain of Hordeum.

SCHOOL OF BREWING,

UNIVERSITY OF BIRMINGHAM. 


\section{$2 \mathrm{BHL}$ Biodiversity Heritage Library}

Brown, Adrian J. 1907. "On the existence of a semi-permeable membrane enclosing the seeds of some of the Gramineae." Annals of botany 21, 79-87. https://doi.org/10.1093/oxfordjournals.aob.a089126.

View This Item Online: https://www.biodiversitylibrary.org/item/235747

DOI: https://doi.org/10.1093/oxfordjournals.aob.a089126

Permalink: https://www.biodiversitylibrary.org/partpdf/318863

\section{Holding Institution}

Smithsonian Libraries

\section{Sponsored by}

Biodiversity Heritage Library

\section{Copyright \& Reuse}

Copyright Status: Not in copyright. The BHL knows of no copyright restrictions on this item.

This document was created from content at the Biodiversity Heritage Library, the world's largest open access digital library for biodiversity literature and archives. Visit BHL at https://www.biodiversitylibrary.org. 